\title{
METACOGNICIÓN: UN FENÓMENO ESTRATÉGICO PARA LA ENSEÑANZA Y EL APRENDIZAJE
}

\author{
METACOGNITION: A STRATEGIC PHENOMENON FOR TEACHING \\ AND LEARNING \\ Darlos Fernando Vélez Gutiérrez ${ }^{*}$, (D) Francisco Javier Ruíz Ortega ${ }^{1}$ \\ velez.carlosf@gmail.com; francisco.ruiz@ucaldas.edu.co \\ ${ }^{1}$ Universidad de Caldas, Caldas, Colombia
}

*Correspondencia: Carlos Fernando Vélez Gutiérrez. Email: velez.carlosf@gmail.com

Recibido: 08.12.2020 | Aprobado: 19.09.2020

\section{RESUMEN}

La discusión sobre la metacognición, un fenómeno cognitivo de reciente caracterización, indispensable en los procesos educativos y la resolución de diferentes problemáticas, se ubica, particularmente, en dos campos disciplinares: la psicología evolutiva y el procesamiento de información; de allí se desprenden perspectivas teóricas que enfatizan en fenómenos como: la teoría de la mente, la evolución de la inteligencia, la sensación del saber, la comprensión, el conocimiento y la regulación. Como conclusiones, para el docente, destacamos la importancia de hacer conscientes las formas de procesamiento mental que realizan los estudiantes como posibilidad de adecuar las estrategias de enseñanza, un requerimiento clave para garantizar que el aprendizaje y el desarrollo sucedan y que el estudiante pueda configurar un rol más efectivo.

Palabras clave: Cognición, metacognición, enseñanza y aprendizaje.

\section{ABSTRACT}

The discussion about metacognition, a recently characterized cognitive phenomenon, indispensable in education processes and problem-solving, falls within two particular fields of knowledge, i.e., evolutionary psychology and information processing. From these fields come the theoretical perspectives that emphasize phenomena such as the theory of the mind, the evolution of intelligence, the sense of knowledge, understanding, knowledge, and regulation. As conclusions, for the teacher, we emphasize the importance of raising awareness about students' mental processing manners as a possibility to adapt teaching strategies, an essential requirement to ensure that learning and development occur and that the student engages more effectively.

Keywords: Cognition, metacognition, teaching, learning. 


\section{INTRODUCCIÓN}

En este artículo revisamos distintas perspectivas teóricas sobre la metacognición, un fenómeno cognitivo que ha cobrado relevancia en las últimas cuatro décadas. Si bien es un fenómeno recientemente caracterizado por la comunidad de psicólogos, especialmente por su trabajo con niñas y niños en los Estados Unidos, se está constituyendo en un componente importante de investigación, reflexión y práctica en diferentes contextos y procesos sociales que incluyen a jóvenes, adultos y ancianos (Frith and Frith, 1999; Briñol y DeMarree, 2012; Frith, 2012; Zohar \& Barzilai, 2013; Chekwa, McFadden, Divine \& Dorius, 2015; Campo, Escorcia, Moreno \& Palacio, 2016; Bueno, 2017).

Flavell $(1979,1987)$ acuñó la noción “metacognición” para referirse al conocimiento que tenemos sobre nuestros propios procesos cognitivos. La cita siguiente transcribe la que, tal vez, fue la primera formulación publicada en una revista especializada:

Studies suggest that young children are quite limited in their knowledge about cognitive phenomena -or in their metacognition- and do relatively little monitoring of their own memory, comprehension, and other cognitive enterprises. Metacognitive knowledge is one's stored knowledge or beliefs about oneself and others as cognitive agents, about tasks, about actions or strategies, and about how all these interact to affect the outcomes of any sort of intellectual enterprise. Metacognitive experiences are conscious cognitive or affective experiences that occur during the enterprise and concern any aspect of it-often, how well it is going. Research is needed to describe and explain spontaneous developmental acquisitions in this area and find effective ways of teaching metacognitive knowledge and cognitive monitoring skills. (Flavell, 1979, p. 906)

En esta reflexión inicial, Flavell identificó como componente fundamental el conocimiento, entendido como lo que saben los niños sobre los fenómenos cognitivos, es decir, sobre lo que ocurre en sus mentes. En segundo lugar, afirmó que este conocimiento, que denominó “metacognition”, está relacionado con el monitoreo que hacen los niños sobre su memoria, su comprensión y otras tareas de la mente. En tercer lugar, reconoció que este conocimiento, que podemos conservar o almacenar, se refiere a nosotros mismos y a las demás personas como "agentes metacognitivos", sobre tareas, acciones y estrategias, así como sus relaciones, para incidir en los resultados de cualquier tipo de actividad intelectual. En cuarto lugar, introdujo el término "experiencias metacognitivas" para referirse a las experiencias cognitivas y afectivas conscientes que surgen en la realización de estas actividades intelectuales. Por último, en quinto lugar, recomendó hacer investigación sobre estos fenómenos cognitivos (metacognición, agencia metacognitiva y experiencia metacognitva) para describir y explicar cuándo los niños los adquieren espontáneamente y cómo enseñar el conocimiento metacognitivo y las habilidades de monitoreo de nuestra actividad cognitiva.

En síntesis, podemos decir que la metacognición, desde estas primeras propuestas teóricas de Flavell, se refiere al conjunto de conocimientos, emociones y sentimientos sobre lo que sucede en nuestras mentes y que adquirimos en nuestra infancia; por esto, también podemos decir que la metacognición es la consciencia que logramos sobre nuestras diversas actividades mentales (operaciones intelectuales, procesamientos de informción y emociones) y las de las personas con las que interactuamos, o el aprendizaje 
que logramos sobre los diversos procesos y productos que configuran nuestras mentes y las de los demás. De ello se desprende que este aprendizaje lo podemos profundizar (a medida que crecemos y nos desarrollamos), lo podemos monitorear y lo podemos enseñar a otras personas (Wilson \& Bai, 2010; Ramakrishnan \& Annakodi, 2013).

\section{Aspectos complementarios}

Si bien disponemos de un número significativo de revisiones y handbooks (Weinstein \& Mayer, 1985; Wellman, 1985; Hertzog \& Dixon, 1994; Schraw \& Moshman, 1995; Flavell, 2004; Frith, 2012; Zohar \& Barzilai, 2013), para este artículo recurrimos especialmente al trabajo de Crespo (2000), que integra claramente las relaciones conceptuales del núcleo de investigación y reflexión sobre metacognición. Esta investigadora chilena identificó 4 perspectivas teóricas y dos campos disciplinares: la psicología evolutiva y el procesamiento de información. En relación con la psicología evolutiva, las dos perspectivas son la teoría de la mente y la evolución de la inteligencia. Por su parte, en relación con el procesamiento de la información, una se refiere a la memoria ("Feeling of Knowing") y otra a la comprensión de lectura (conocimiento y regulación). A continuación, exponemos los planteamientos centrales de estas concepciones.

"La teoría de la mente" surge de los estudios de Piaget $(1985 ; 2000)$ en las décadas de los 70 y los 80 , sobre los primeros años de la infancia, cuando se da cuenta de que este tipo de conocimiento sobre el pensamiento de los demás no ha sido diferenciado teóricamente. Sus propuestas son actualmente rebatidas por otros investigadores de la psicología evolutiva; esto quiere decir que no hay consenso: algunos están de acuerdo con Piaget y otros sólo parcialmente.

Estas tensiones al interior de las comunidades que investigan el desarrollo cognitivo de los niños generaron dos subcorrientes (o perspectivas teóricas) al interior de las comunidades académicas interesadas en la teoría de la mente: la teoría de la mente como simulación (los niños se dan cuenta de que conocen su mente y por eso le asignan esta misma habilidad a los otros niños) y la teoría de la mente como construcción mental (los niños la construyen a medida que observan e interactúan con otros niños y con personas adultas). A la base de estas dos perspectivas, hay una preocupación común: ¿En qué momento y cómo los niños diferencian y relacionan lo que sucede en su interior con lo que ocurre en el mundo que los rodea? Una primera diferenciación que apunta a resolver esta pregunta tiene que ver con los contenidos del "conocimiento metacognitivo" (no con la metacognición): este conocimiento está constituido por 5 subtipos de conocimiento (Wellman 1985):

\section{Existencia}

Sabemos que en nuestro cerebro/mente ocurren diferentes fenómenos, suceden distintas cosas (operaciones, procesos) y, sobre todo, diferenciamos lo que ocurre en nuestra mente de lo ocurre fuera de ella; por ejemplo: distinguimos entre lo que recordamos y lo que hacemos a partir de ese recuerdo. A veces, sentimos rabia, pero damos una caricia, o nos da temor, pero nos comportamos como valientes. En pocas palabras, son cosas (fenómenos, procesos, operaciones, actividades) distintas lo que pensamos de lo que hacemos; a veces, hay coherencia y otras incoherencias entre estos dos tipos de fenómenos: internos y externos. 


\section{La distinción de los procesos}

Además del conocimiento (la convicción, la sensación, la consciencia) sobre la diferencia entre lo que ocurre en nuestra mente de lo que ocurre por fuera de nuestro cuerpo, también nos damos cuenta de que lo que ocurre en nuestra mente es plural (es diverso); es decir, hay distintos fenómenos (funciones, procesos, operaciones, actividades) mentales. Podemos diferenciar cuando "sabemos" de cuando "imaginamos" o "adivinamos", cuando "repetimos" de cuando "comprendemos", cuando "decimos la verdad" de cuando "mentimos", cuando "tenemos certeza" de cuando "dudamos", etc.

\section{La integración}

Igualmente, sabemos que estos distintos tipos de actividad cognitiva los podemos relacionar entre sí; se pueden combinar de múltiples maneras, según sea la situación y las intenciones que tengamos cuando decidimos utilizar una u otra. Podemos seleccionarlos, separarlos o agruparlos según nuestra voluntad; por supuesto, en casos normales, porque es evidente que en situaciones patológicas se puede distorsionar una actividad por otra.

\section{Conocimiento de las variables}

Como mencionamos en el punto anterior, la selección de una actividad cognitiva sobre otra obedece al conocimiento de las variables que diferencian la tarea o la estrategia que utilizaremos. Por ejemplo, es más sencillo aprenderse un nombre corto que uno largo, un apellido común en nuestro medio que uno extraño. Recordar el nombre de un medicamento popular y tradicional que el nombre de uno nuevo, etc. Responder por una operación matemática elemental sin utilizar un medio externo que hacerlo con una operación compleja. ¿Cuáles son las características de la actividad cognitiva que tenemos que realizar, cuál es su naturaleza, su grado de dificultad, etc.? Además, la relación que establecemos entre este grado de dificultad de la actividad cognitiva con las habilidades que poseemos para lograrla.

\section{Monitoreo cognitivo}

Somos capaces de monitorear todas estas actividades mentales y sabemos cómo hacerlo; por ejemplo, al tiempo que vamos resolviendo mentalmente la operación matemática vigilamos que la estemos haciendo bien o nos damos cuenta de que hicimos algo mal. Podemos evaluar lo que pensamos antes de decirlo y cambiarlo incluso antes de llegar a decirlo. Sabemos que podemos darnos cuenta de lo que pensamos y que podemos modificarlo.

Como es evidente, ésta es otra forma de clasificar el conocimiento metacognitivo. A diferencia de lo que expusimos anteriormente, aquí el conocimiento metacognitivo sobre la persona se categoriza para hacer algunas distinciones que consideramos complementarias. Esto es así porque es la preocupación central de la teoría de la mente: conocer los contenidos de nuestro conocimiento sobre nuestra mente y la mente de los demás (Tomasello, 2003).

Ahora bien, Perner (1994) coincide con Wellman sobre las etapas que vive el niño hasta llegar al desarrollo metacognitivo, pero lo ve de una manera más compleja y propone la distinción entre representaciones primarias, secundarias y meta-representaciones; es decir, el niño llega a modelar lo que ha modelado sobre sus percepciones: de las percepciones a los modelos y de estos modelos a unos meta-modelos. En palabras de 
Crespo (2000), "comprender la relación representacional entre el medio simbólico y lo representado" (p. 100).

Para Perner (1994), "los informes sobre estados mentales - en particular, creencias- son metarrepresentaciones desde el momento en que son informes (representaciones) de representaciones mentales" (p. 76). Una cosa es percibir y otra recordar lo percibido, para luego valorar, pensar, reflexionar, problematizar esos recuerdos. Estas transformaciones no son cuantitativas sino cualitativas; por eso, existe la posibilidad de convertir esta capacidad en habilidad, competencia o experticia (Acosta y Vasco, 2013).

Si bien Wellman (1985) homologa teoría de la mente con metacognición, hay otros autores que no están de acuerdo. Parece que la diferencia fundamental tiene que ver con el lugar que juega la regulación (la autorregulación) que no es mencionada por la teoría de la mente: una cosa es saber lo que ocurre en mi mente para entender lo que sucede en la mente de los demás y otra es, a partir de este conocimiento, de este grado de consciencia o darse cuente de, regular mi propia actividad cognitiva, para mejorarla, para transformarla, en función de una tarea o una situación social particular, como la relación de enseñanza y aprendizaje.

De todas maneras, Flavell coincide en que son perspectivas cercanas que tienen elementos comunes; incluso afirma que el trabajo sobre metacognición es una "segunda ola" dentro del desarrollo teórico y la investigación sobre teoría de la mente que inicia Piaget (1985; 2000). En esta tradición menciona como autores más representativos a "Brown, Bransford, Ferrara, and Campione (1983), Flavell, Miller, and Miller (2002), Kubn (1999), Moshman (1998), and Schneider and Bjorklund (1998)" (Flavell, 2004, p.275).

En ninguno de estos estudios se observan referencias a las nociones que el sujeto pueda tener sobre las capacidades involucradas en una tarea cognitiva, la interpretación y vivencia de experiencias metacognitivas, ni el uso de recursos estratégicos para mejorar su actuación”. (Crespo, 2000, p.101)

Además de la teoría de la mente, Crespo (2000) menciona que el conocimiento metacognitivo es una tendencia de desarrollo cognitivo de la adolescencia. En esta dirección, Flavell prefiere no hablar de estadios, como sí lo hacía Piaget; habla de tendencias porque no está de acuerdo con la propuesta de progresividad de la madurez cognitiva de las personas que expone Piaget. Las siete tendencias que propone Flavell son (Crespo, 2000, p. 102):

1) Incremento en la capacidad de procesamiento de la información;

2) Aumento de conocimiento en dominios especificos;

3) Presencia de algunos rasgos propios de las operaciones concretas y formales

4) Predisposición más cuantitativa y orientada a la medida;

5) Un sentido del juego mental y una capacidad de juggar su nivel de corrección;

6) Desarrollo metacognitivo (como conocimiento y como experiencia) y

7) Habilidad de mejorar competencias ya existentes, pero todavia inmaduras.

Flavell tampoco está de acuerdo con atribuirle a las operaciones formales la etapa final del desarrollo cognitivo de las personas: "un hombre con una mente totalmente científica que 
aborda racionalmente los diferentes aspectos de su quehacer, no parece sostenerse si consideramos las actitudes, pensamientos y reflexiones de los individuos a lo largo de la vida adulta" (p. 101).

Desde esta perspectiva, podemos decir que la metacognición es apenas una parte del desarrollo ontogenético de las personas, de su historia personal, y que, además, se relaciona con otros fenómenos cognitivos en el marco de unas tendencias del desarrollo y no como una etapa precisa y distinta, aislada de otras, que retroalimentan todo el proceso cognitivo a lo largo de la vida (Karmiloff-Smith, 1992).

Crespo, además, menciona otros autores (Baker, 1989 y Chi, 1987) que no están de acuerdo con la forma como se entiende la metacognición en el proceso de desarrollo. Estos autores afirman que el conocimiento experto (lo que podríamos llamar conocimiento dominio específico) favorece la actuación y la regulación metacognitiva de las personas y que, por tanto, es muy importante identificar qué hace la diferencia en la resolución de las tareas: el conocimiento metacognitivo o el conocimiento experto asociado con ellas.

De todas maneras, estas distinciones de la comunidad de psicólogos evolucionistas aclaran un poco más los planteamientos iniciales que hacíamos sobre metacognición: una cosa es una estrategia cognitiva y otra una estrategia metacognitiva. Las estrategias cognitivas actúan sobre la tarea y los objetivos relacionados con su realización, mientras que las estrategias metacognitivas se refieren a controlar las estrategias cognitivas. Es este tipo de relación el que nos permitió ubicar a las estrategias metacognitivas en otro nivel.

Para terminar su revisión, Crespo (2000) expone las dos líneas de investigación que surgen desde la lógica del procesamiento de información: Feeling of knowing y conocimiento y regulación en tareas de comprensión lectora.

Feeling of Knowing, que traducimos como "la sensación de saber", es una evidencia cognitiva que muestra que, cuando procesamos información, corregimos los errores o dificultades que se nos presentan como si tuviéramos un sistema de monitoreo cognitivo durante dicho procesamiento que nos indica que hay algo más que sabemos o podemos hacer. Este control ejecutivo, o sistema ejecutivo, es la función cognitiva que le permite a nuestra mente funcionar como un "termostato" que, al parecer, precisa regularse constantemente hasta alcanzar un estado de equilibrio, como lo describió Piaget.

El fenómeno más estudiado desde esta perspectiva es denominado "de la punta de la lengua" y expresa metafóricamente esa sensación de saber. Cuando esto nos pasa, a propósito del algún olvido de la memoria, decimos "lo tengo en la punta de la lengua". En otras palabras, sabemos que sabemos sobre el contenido de información que estamos procesando, pero no recordamos en el momento que es necesario exteriorizar ese conocimiento. "Dicho mecanismo de control estaría activado en forma constante pero se volvería evidente -bajo la forma del Feeling of Knowing-cuando el proceso de recuperación no ocurre" (Crespo, 2000, p. 107).

Estas evidencias son las que llevaron a postular la existencia de dos niveles cognitivos: uno que tiene que ver con el objeto, por ejemplo, recordar una secuencia de nombres que es preciso verbalizar, y otro que tiene que ver con el procesamiento mental que orienta la realización de dicha actividad, por ejemplo, identificar qué estrategia de 
recordación funcionará mejor y cuál no; por dónde empezar a recordar y cómo continuar la tarea de recuperación de dicha información.

Entre ambos niveles bay un flujo de información en el cual el metanivel ejerce un papel de dominancia o control respecto del nivel de objeto. El nivel objeto, por su parte, envía información al nivel meta sobre su situación actual a través del monitoreo (Crespo, 2000, p. 107).

Como podemos observar, es claro que un planteamiento como éste está muy cerca de las conceptualizaciones sobre metacognición, particularmente la afirmación de la existencia de dos niveles cognitivos que intervienen sobre contenidos diferentes que interactúan. Incluso Crespo (2000) clasifica "la sensación de saber" como una manifestación muy específica de la metacognición, así sus promotores no estén muy interesados en adscribirse explícitamente a esta escuela de pensamiento. Su trabajo de investigación está enfocado en la memoria (la metamemoria) y, por tanto, no tienen los mismos intereses que la comunidad que va más allá de esta preocupación; adicionalmente, no tienen en cuenta el papel de la consciencia o la existencia de una intencionalidad en la interacción entre estos niveles. Ellos tratan este fenómeno como un mecanismo mental que no tiene una relación directa con las características sociales o culturales de las personas; es un procesamiento funcional de la información y, por ello, no es necesario vincularlo con la experiencia personal de quien lo realiza.

Por último, en relación con conocimiento y regulación de la comprensión lectora, las autoras más reconocidas son Baker y Brown (1984) quienes afirman que...

... the ability to use self-regulatory mechanisms to ensure the successful completion of the task, such as checking the outcome of any attempt to solve the sêpiproblem, planning one's next move, evaluating the effectiveness of any attempted action, testing, and revising one's strategies for learning, and remediating any difficulties encountered by using compensatory strategies (p. 22).

Esto significa que disponemos de mecanismos cognitivos de autorregulación que podemos usar para enfrentar una tarea como la comprensión lectora. Algunos mecanismos de autorregulación son: verificación del resultado que obtenemos por cada intento de solución que elegimos, planeación de la siguiente actividad que realizaremos, evaluación de la efectividad de todas las acciones puestas en marcha, prueba y revisión de las estrategias de aprendizaje propias y resolución de las dificultades que surjan mediante el uso de estrategias compensatorias de los intentos fallidos.

Estos mecanismos, por tanto, articulan los saberes declarativo y procedimental en dos grandes fases; por un lado, el conocimiento metacognitivo y, por el otro, la regulación metacognitiva. Brown (citada en Crespo, 2000) diferencia estas fases al reconocer que el conocimiento metacognitivo es más estable, mientras la regulación metacognitiva varía dependiendo de la tarea. En este sentido, afirma que el conocimiento metacognitivo es utilizado reiteradamente por las personas, puede aumentar con la edad, volverse más complejo y ser utilizado en la resolución de diferentes tipos de tareas. En pocas palabras, a medida que conocemos nuestros procesos cognitivos, los podemos profundizar y complejizar, para utilizarlos cuando sean necesarios; en ello radica su estabilidad: los tenemos a nuestra disposición desde el momento que los adquirimos. Por su parte, en relación con la regulación metacognitiva, depende de los factores externos que involucra 
la tarea; cada tarea contiene sus propias demandas de conocimiento metacognitivo y, por tanto, esto la hace una fase más inestable y contingente.

La estabilidad o inestabilidad de estas dos fases también están relacionadas con la posibilidad de referirnos a ellas. El conocimiento metacognitivo puede ser expresado en palabras, mientras que la regulación metacognitiva con frecuencia sucede inconscientemente, automáticamente, y sólo es expresable cuando surge una dificultad que requiere un abordaje intencional, consciente. Además, afirma que el conocimiento metacognitivo es falible porque puede ser contrastado con criterios de validez que lo vuelven relativo en ciertas circunstancias. Por último, asegura, en coherencia con los autores más representativos del campo, que este conocimiento aparece en la niñez y se fortalece en la edad adulta.

De otro lado, Crespo (2000) reconoce que, si bien hay muchas semejanzas con los planteamientos clásicos de Flavell sobre metacognición, hay algunas diferencias significativas: En primer lugar, Brown separa conocimiento y regulación, mientras Flavell afirma que conocimiento declarativo y conocimiento procedimental hacen parte de un todo y no son fases discretas. En segundo lugar, para Brown, la regulación metacognitiva es independiente de la edad, mientras que Flavell y sus seguidores están convencidos de su emergencia en la adolescencia y su posterior fortalecimiento en la edad adulta: es la experiencia la que contribuye a su desarrollo. En tercer lugar, aunque Brown y Flavell se inscriben en la misma tradición piagetiana, sus énfasis sobre la autorregulación no son los mismos: Brown entiende la autorregulación en función de la resolución de la tarea, de la efectividad de las acciones que la persona decida utilizar, mientras que Flavell la entiende más en función de la regulación de los procesos cognitivos, de las actividades cognitivas involucradas en la tarea y no en la tarea misma.

Para terminar, Crespo (2000) subraya tres grandes conclusiones:

1. La metacognición como fenómeno cognitivo y como objeto de estudio articula un conjunto "diverso y multifacético" de variables y actividades cognitivas que son parte de nuestra mente, que configuran nuestro pensamiento y nuestras emociones/sentimientos, que surgen en la adolescencia y se consolidan en la edad adulta. Su importancia radica en la posibilidad de autodirigir nuestra actividad cognitiva, necesaria para enfrentar conscientemente (aunque también automáticamente) una multiplicidad de tareas de diverso tipo.

2. Su trayectoria histórica está relacionada con diversos autores, en la filosofía y en las ciencias cognitivas, aunque más recientemente, se vinculan otras disciplinas como la didáctica de las ciencias, la neurofisiología y la psiquiatría; sin embargo, no hay consenso en la delimitación conceptual del fenómeno y su caracterización como constructo teórico.

3. Recomienda acoger la propuesta de Hertzog y Dixon (1994) quienes prefieren entender la metacognición como un "grupo de constructos interrelacionados que corresponden a una única noción central de cognición sobre la cognición"; esta noción central es especificable si distinguimos entre dominio cognitivo (por ejemplo, memoria, resolución de problemas, atención y comprensión) y tipos de cognición posibles en cada dominio (por ejemplo, representaciones, creencias y experiencias). En sus 
palabras: "La idea es aceptar la complejidad de lo metacognitivo, tratando de rescatar su riqueza y sus posibilidades de aplicación” (p. 112).

\section{CONCLUSIONES}

Como podemos ver, aquí subyacen preocupaciones sobre fenómenos claramente diferenciados que se van integrando en una teoría común, sobre preocupaciones teóricas e investigativas que van confluyendo, incluso desde campos disciplinares con orígenes diversos e intereses aparentemente inconexos. ¿Cuáles son estos fenómenos? Por un lado, la teoría de la mente, es decir la consciencia sobre la existencia de la mente del otro, la habilidad de asignarle a otra persona habilidad (o capacidad) de pensamiento, de tener intenciones, de ser inteligente, de razonar; también, la interacción mental que ocurre en cada uno de nosotros (entre nuestras actividades cognitivas) y con las actividades cognitivas de los demás y las relaciones que hay entre pensamiento y comportamiento: ciertos pensamientos o actividades mentales generan actividades o conductas particulares, cognitivas, metacognitivas, emotivas o motrices. Por otro lado, el desarrollo de la inteligencia (¿una inteligencia o múltiples inteligencias?), el desarrollo mental de los seres humanos y los cambios que ocurren en nuestra mente (cerebro) a medida que maduramos, que crecemos, que los niños se convierten en adolescentes, jóvenes y adultos. En síntesis, la maduración de la mente, el desarrollo cognitivo y sociocognitivo, para lo cual se han identificado y descrito fenómenos como los mencionados en esta reflexión sobre la literatura especializada.

Por último, si en las primeras décadas de investigacion y reflexión sobre la metacognición su énfasis estuvo en la caracterización del funcionamiento de la memoria de los niños, utilizando técnicas experimentales, los desarrollos teóricos y metodológicos más recientes giran hacia una perspectiva social de la metacognición, con niños, jovenes y adultos, en la que el contexto y las situaciones in vivo cobran especial relevancia (Goos, Galbraith, and Renshaw, 2002; Duman, 2006; Schwartz, Chase, Chin, Oppezzo, Kwong, Okita, Biswas, Roscoe, Jeong and Wagster, 2009; Briñol \& DeMarree, 2012; Shea, Boldt, Bang, Yeung, Heyes, and Frith, 2014; Van De Bogart, Dounas-Frazer, Lewandowski and Stetzer, 2017; Tullis \& Fraundorf, 2017). Desde estos planteamientos, la metacognición se convierte en una construcción social que impacta a los individuos y su entorno; de allí su relevancia en las dinámicas educativas de todos los niveles, como lo propuso Vygotsky (2000) en su teoría sociocultural del desarrollo cognitivo.

\section{REFERENCIAS BIBLIOGRÁFICAS}

Acosta, D.A., Vasco, C.E. (2013). Habilidades, competencias y experticias: más allá del saber qué y el saber cómo. Bogotá: Unitec, CINDE, Universidad de Manizales.

Baker, L. (1989). Metacognition, Comprehension Monitoring and the Adult Reader. Educational Psychology Review, 1(1), 3-38.

Baker, L. \& Brown, A. L. (1984). Cognitive monitoring in Reading. In Flood, J. (Eds.) Understanding Reading Comprehension: Cognition, Language and the Structure of Prose. Delaware: I.R.A. (pp. 21-43). 
Briñol, P. y DeMarree, K. G. (2012). Social Metacognition. New York: Psychology Press Taylor \& Francis Group.

Bueno, D. (2017). Neurociencia para educadores. Barcelona: Octaedro.

Campo, K., Escorcia, D., Moreno, M. \& Palacio, J. (2016). Metacognición, escritura y rendimiento académico en universitarios de Colombia y Francia. Avances en Psicología Latinoamericana, 34(2), 233-252.

Chekwa, E., McFadden, M., Divine, A. \& Dorius, T. (2015). Metacognition: Transforming the Learning Experience. Journal of Learning in Higher Education, 11(1), 109-112.

Chi, M. (1987). Representing knowledge and metaknowledge: implications for interpreting metamemory research. In Weinert, F. y Klowe, R. (Eds.). Metacognition, Motivation and Understanding. Broadway: Lawrence Erlbaum Associates, Inc. (pp. 239-264).

Crespo, N. M. (2000). La Metacognición: Las diferentes vertientes de una Teoría. Revista Signos, 33(48), 97-115.

Damasio, A. (1999). El error de Descartes. La emoción, la razón y el cerebro humano. Santiago de Chile: Crítica - Editorial Andres Bello.

Duman, B. (2006). The effect of brain-based instruction to improve on students' academic achievement in social studies instruction. 9. International Conference On Engineering Education San Juan, Puerto Rico July 23-28. ICEE-2006.

Flavell, J. H. (1979). Metacognitive and Cognitive Monitoring: A New Area of CognitiveDevelopmental Inquiry. American Psychologyst, 34, 906-911.

Flavell, J.H. (1987). Speculations about the nature and development of metacognition. In F. Weinert, \& R. Kluwe (Eds.), Metacognition, motivation and understanding (pp. 21-29). Hillsdale, NJ: Erlbaum.

Flavell, J. H. (2004). Theory-of-Mind Development: Retrospect and Prospect. MerrillPalmer Quarterly, 50(3), 274-290.

Frith, C. D. and Frith, U. (1999). Interacting Minds - A Biological Basis. Cognitive Psychology, 286 (26) November.

Frith, C. D. (2012). The role of metacognition in human social interactions. Phil. Trans. R. Soc. B 367, 2213-2223.

Goos, M.; Galbraith, P.; and Renshaw, P. (2002). Socially mediated metacognition: Creating collaborative zones of proximal development in small group problem

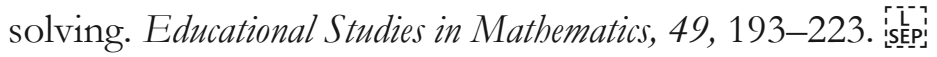

Hertzog, C., \& Dixon, R. (1994). Metacognitive development in adulthood and old age. In J. Metcalfe \& A. Shimamura (Eds.), Metacognition: Knowing about knowing (pp. 227 - 251). Cambridge, MA: Bradford.

Karmiloff-Smith, A. (1992). Auto-organización y cambio cognitivo. Substratum, 1(1), 1943. 
Perner, J. (1994). Comprender la mente representacional. Barcelona: Paidós Ibérica

Piaget, J. (1985). La toma de conciencia. Madrid: Ediciones Morata S.A. (Tercera edición).

Piaget, J. (2000). La equilibración de las estructuras cognitivas. Problema central del desarrollo. México: Siglo XXI Editores. (Sexta edición en español).

Ramakrishnan, J. \& Annakodi, R. (2013). Brain based learning strategies. International Journal of Innovative Research and Studies (IJIRS), 2(5), 235-242.

Schraw, G. and Moshman, D. (1995). Metacognitive Theories. Educational Psychology Review, $7(4)$.

Shea, N., Boldt, A., Bang, D., Yeung, N., Heyes, C. and Frith, C. D. (2014). Supra-personal cognitive control and metacognition. Trends in Cognitive Sciences, 18(4).

Schwartz, D. L., Chase, C., Chin, D. B., Oppezzo, M., Kwong, H., Okita, S., Biswas, G., Roscoe, R., Jeong, H., and Wagster J. (2009). Interactive Metacognition: Monitoring and regulating a teachable Agent. In: Handbook of metacognition in education. New York: Routledge.

Tomasello, M. (2003). The Cultural Origins of Human Cognition. Cambridge, Mass: Harvard University Press.

Tullis, J. G. and Fraundorf, S. H. (2017). Predicting others' memory performance: The accuracy and bases of social metacognition. Journal of Memory and Language, 95(1), 124-137.

Van De Bogart, K.L.; Dounas-Frazer, D.R.; Lewandowski, H.J. and Stetzer,

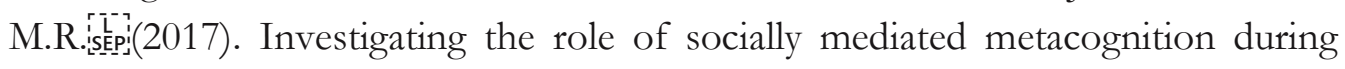
collaborative troubleshooting of electric circuits. Physical Review Physics Education Research,13(2).

Vygotsky, L. (2000). El desarrollo de los procesos psicológicos superiores. Barcelona: Crítica. Primera edición en Biblioteca de Bolsillo.

Wellman, H. M. A. (1985). The Origins of Metacognition. En Forrest Presley, D. L., Mackinton, G. E. and Waller, T.G. (Eds.) Metacognition, cognition and Human Perfomance, Orlando: Academic Press (pp.1-30).

Wilson, N.S., Bai, H. (2010). The relationships and impact of teachers' metacognitive knowledge and pedagogical understandings of metacognition. Metacognition Learning 5, 269-288

Zohar A. \& Barzilai, S. (2013) A review of research on metacognition in science education: current and future directions. Studies in Science Education, 49(2), 121-169. enseñanza y el aprendizaje. Puriq, 3(1), 93-103. https://doi.org/10.37073/puriq.3.1.112 